\title{
Lymphatic transport of high-density lipoproteins and chylomicrons
}

\author{
Gwendalyn J. Randolph ${ }^{1}$ and Norman E. Miller ${ }^{2}$ \\ 1Department of Pathology and Immunology, Washington University School of Medicine, St. Louis, Missouri, USA. \\ ${ }^{2}$ Magdalen College, Oxford University, Oxford, United Kingdom.
}

\begin{abstract}
The life cycles of VLDLs and most LDLs occur within plasma. By contrast, the role of HDLs in cholesterol transport from cells requires that they readily gain access to and function within interstitial fluid. Studies of lymph derived from skin, connective tissue, and adipose tissue have demonstrated that particles as large as HDLs require transport through lymphatics to return to the bloodstream during reverse cholesterol transport. Targeting HDL for therapeutic purposes will require understanding its biology in the extravascular compartment, within the interstitium and lymph, in health and disease, and we herein review the processes that mediate the transport of HDLs and chylomicrons through the lymphatic vasculature.
\end{abstract}

HDLs constitute one of four major classes of particles that transport lipids between organs, tissues, and cells (Table 1). "Good cholesterol," the layman's term for HDL cholesterol, makes reference to the established role of HDL in transport of cholesterol from peripheral tissues to plasma, and from plasma to the liver, where the cholesterol is processed for packaging into bile acids for excretion. This putatively anti-atherogenic (and hence "good") biological role of HDL in supporting excretion of excess cholesterol was first postulated by Glomset and colleagues (1) and has since been termed reverse cholesterol transport (2). Subsequently, significant evidence identified HDL as a therapeutic target to combat atherosclerosis. However, recent clinical trials designed to test whether increasing plasma HDL levels is therapeutically beneficial did not demonstrate efficacy (3-5), and genetic studies have refuted the prediction that higher levels of plasma HDL in humans associates with protection from cardiovascular disease (6). Harnessing the role of HDL in cholesterol clearance remains of great interest, but the field now recognizes that simple measurements of plasma HDL cholesterol do not capture its ability to support cholesterol efflux from cells, including cholesterol-loaded macrophages within atherosclerotic plaques. Indeed, the most important site from which HDLs act to promote cholesterol efflux from cells is in the extracellular matrix of tissues, not in plasma. It is plausible that some persons who display high levels of plasma HDL cholesterol have poor HDL recirculation and/or function within the interstitium of tissues, leading to poor cholesterol efflux at relevant sites. This Review considers what is known about HDL cholesterol in the interstitium and its transport through lymphatic vessels as a requisite part of its role in reverse cholesterol transport. To more comprehensively consider this topic, we also discuss chylomicron transport via lymphatics, a process that may provide insight into transport of HDL from other peripheral tissues.

\section{Transit of HDL through the interstitium and lymph}

The major apolipoprotein of HDL is apoA1. When the total volume of interstitial fluid is taken into account and its concentration therein considered, approximately half of all apoA1 in

Conflict of interest: Norman E. Miller is a consultant to uniQure BV, Amsterdam, Netherlands.

Citation for this article: J Clin Invest. 2014;124(3):929-935. doi:10.1172/JCI71610. the body is extravascular and found within interstitial fluid of peripheral organs (7). Calculations based on lipoprotein particle numbers in plasma (8) and concentrations of apoA1 and apoB (the major protein of LDL) in plasma and peripheral lymph (9) indicate that in normal human interstitial fluid there are usually more than 50 HDL particles to one LDL particle. Nonetheless, in spite of its manifest importance in relation to lipid transport in health and disease, there is a dearth of information on HDL in interstitial fluid of peripheral tissues, owing to the difficulty of obtaining sufficient fluid under physiologic conditions.

Although subcutaneous implantation of microdialysis probes provides valuable information on movement of small molecules between plasma and interstitial fluid in humans, the molecular size cut-off of the probes is too low for recovery of lipoproteins $(10,11)$. Subcutaneous wicks (12) provide insufficient material for analysis of lipoprotein composition. Greater volumes are obtained from suction blisters (13), but suction blister fluid is non-physiologic, formed by separation of the dermis from epidermis (14). The only viable approach is collection of interstitial fluid as it drains from tissues via afferent lymphatic vessels before reaching LNs (Figure 1). An underlying tenet of this approach is that interstitial fluid travels down a pressure gradient from blood to lymphatic capillaries such that the composition of lymph is a close reflection of interstitial fluid itself (15). As observed in mice, a key feature enabling movement of fluid into lymph is the structure of inter-endothelial junctions, organized so that adhesions are discontinuous, like buttons on a coat, to generate flaps between the buttons (Figure 1) that can be pulled open under tension by anchoring filaments attached to the capillary, making the vessel accessible to large macromolecules (16). Transcytosis following uptake of fluid by micropinocytosis and/or receptor-mediated endocytosis may also contribute to entry of lymph into lymphatic capillaries (17).

The first data on human peripheral lymph lipoproteins were obtained by Reichl and colleagues, using fluid collected from a superficial vessel in the dorsum of the foot. In studies conducted between 1973 and 1989, they showed that lymphatic lipoproteins differ from those in plasma in concentration and composition. Concentrations of apoA1 and apoB were much lower than in plasma (18-20), and all apoB was in LDL (21). Studies with radiolabeled lipoproteins confirmed that lymph apolipoproteins were derived from plasma (22). Measurements of cholesterol-specific radioactiv- 


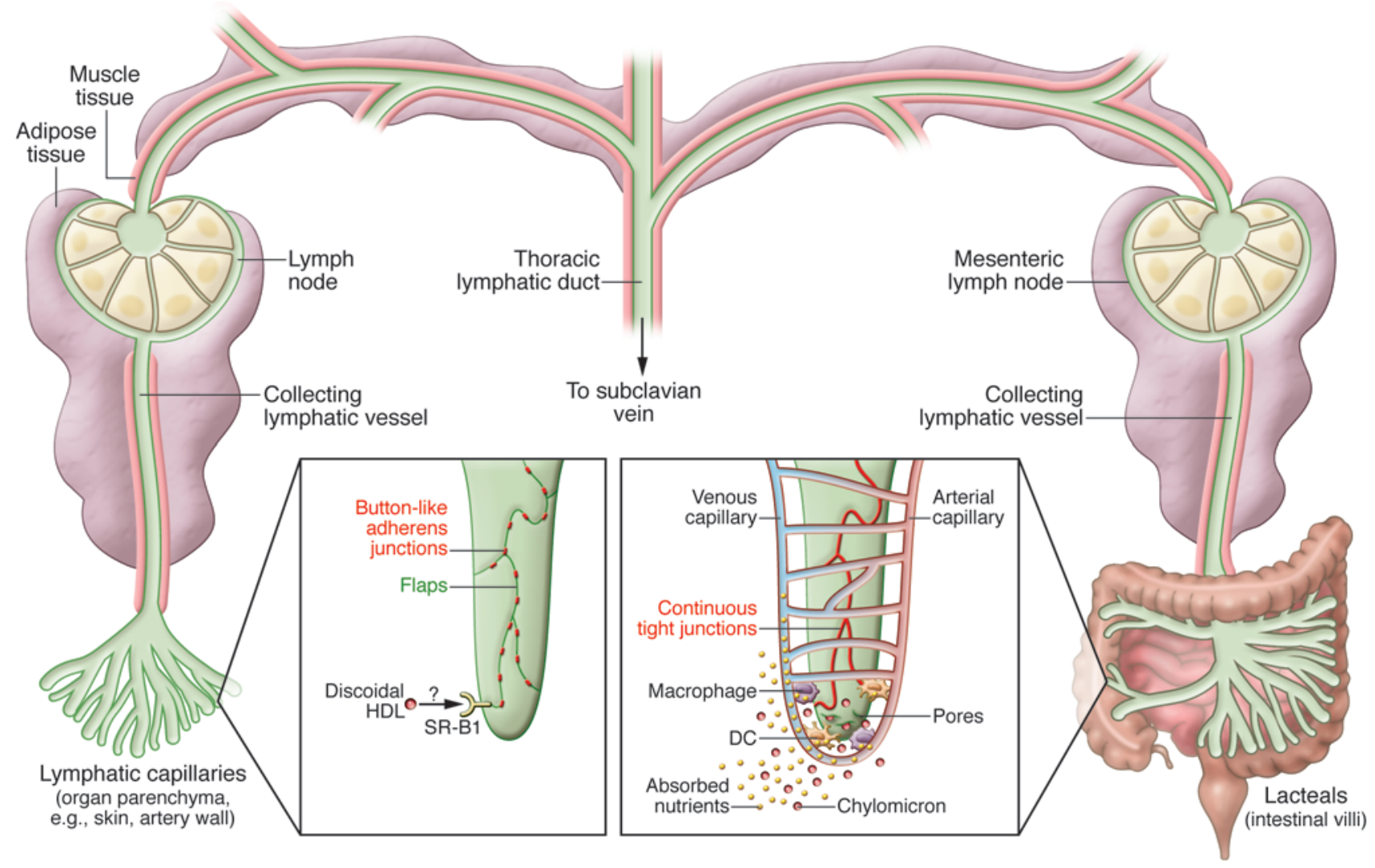

\section{Figure 1}

Organization of the lymphatic vasculature. Lymphatic capillaries form blind-ended vessels in all organs (referred to as lacteals in the intestine). These vessels converge and transition into collecting lymphatic vessels that are surrounded by muscle (red lines overlaying lymphatic vessels). Collecting vessels are interrupted by LNs. Collecting vessels and the LNs are surrounded by adipose tissue. The largest collecting vessel, the thoracic duct, drains lymph collected from all organs into the bloodstream at the subclavian vein. Insets detail the structure of lymphatic capillaries with respect to lipoprotein absorption. In tissues such as skin and lung, button-like junctions separate endothelial cells and create flaps, which allow receptor-independent entry of molecules into lymph. In intestine, large pores may form at the tips. The precise artery-capillary organization is unknown. Entry of discoidal HDL into skin lymph may be mediated by receptors through binding of SR-B1 (left inset), but more work is needed to confirm this. The right inset depicts the fenestrated transition from arterial capillary to venous blood capillary around each intestinal lacteal. Smaller nutrients not packaged in chylomicrons enter the blood vasculature that leads to the portal vein, but chylomicrons are too large. They enter the lacteal vessels by means of size exclusion, which also plays a key role in routing of HDL into lymph in other organs. Macrophages and DCs endocytose at least some molecules in intestinal villi, reducing their transit into the lacteal vessels, even if they bypass entry into the blood vasculature.

ity in lymph several weeks after intravenous infusion of radiolabeled cholesterol indicated that this exceeded that of plasma cholesterol (23). apoA1-containing particles had a broader size spectrum in lymph than in plasma and were enriched in cholesterol of relatively high specific radioactivity $(19,24)$. These studies, reviewed elsewhere in detail (25), were consistent with the concept proposed by Glomset (1) that HDLs are the transport vehicles for cholesterol from tissues. Reichl et al. (26) subsequently found that peripheral lymph contains particles with apoA1, but no apoA2, of similar size and charge as pre $\beta$-HDLs, small lipid-poor particles that Castro and Fielding (27) had shown to be the primary acceptors of cell-derived cholesterol from cultured fibroblasts. An intervention study showed that treatment with gemfibrozil, an oral fenofibrate activator of PPAR $\alpha$, increased apoA 1 and cholesterol in lymph but not in plasma and demonstrated the potential of the method for obtaining unique insight into extravascular cholesterol transport (28).

Miller and coworkers (29) improved the lymph collection procedure, based on an original method of Engeset et al. (30), in which lymph is collected from a larger afferent lymphatic vessel in the lower leg, allowing collection at flow rates of $0.25-2.0 \mathrm{ml} / \mathrm{h}$ for several days. The lymph in this vessel is derived from skin, adipose tissue, and connective tissue. Consistent with Reichl et al. (18-21), lymph was essentially devoid of VLDL. With all apoB in LDLs $(9,29)$, the apoA1/apoB and sphingomyelin/phosphatidylcholine ratios were greater than in plasma. Several weeks after infusion of intravenous radiolabeled cholesterol, the specific radioactivity of lymph cholesterol exceeded that of plasma cholesterol (31). The HDLs of lymph were enriched in large apoA1-containing particles with a high content of unesterified cholesterol, phospholipids, and apo $E(7,9,29)$. At the other end of the size spectrum, the smallest apoA1-containing particles were enriched in phospholipid. Lymph also contained discoidal HDL particles (29). Such HDLs are never seen in plasma except in subjects with familial lecithin-cholesterol acyltransferase (LCAT) deficiency. Of particular relevance to cholesterol clearance from the periphery, total cholesterol concentration in lymph HDL was about $30 \%$ greater than could be explained 
Table 1

Major classes of lipoproteins

\begin{tabular}{|c|c|c|c|c|c|c|c|c|c|c|c|}
\hline \multirow[t]{2}{*}{ Class } & \multirow[t]{2}{*}{ Origin } & \multirow[t]{2}{*}{$\begin{array}{l}\text { Major } \\
\text { function }\end{array}$} & \multirow[t]{2}{*}{$\begin{array}{l}\text { Diameter } \\
\quad(\mathrm{nm})\end{array}$} & \multirow[t]{2}{*}{$\begin{array}{l}\text { Density } \\
(\mathrm{g} / \mathrm{ml})\end{array}$} & \multirow[t]{2}{*}{$\begin{array}{l}\text { Major } \\
\text { protein }\end{array}$} & \multirow[t]{2}{*}{$\begin{array}{l}\text { Concentration in } \\
\text { interstitial fluid }\end{array}$} & \multicolumn{5}{|c|}{$\begin{array}{l}\text { Average composition } \\
\text { by weight (\%) }\end{array}$} \\
\hline & & & & & & & TG & CE & PL & Chol & Protein \\
\hline Chylomicrons & Ileum & $\begin{array}{l}\text { Transport ingested } \\
\text { fat and fat-soluble } \\
\text { vitamins }\end{array}$ & $200-1,000$ & $<0.95$ & apoB48 & Absent & 85 & 3 & 8 & 2 & 1 \\
\hline VLDL & Liver & $\begin{array}{c}\text { Transport synthesized } \\
\text { glyceride }\end{array}$ & $30-90$ & $0.95-1.006$ & apoB100 & Absent & 55 & 18 & 20 & 5 & 9 \\
\hline LDL & $\begin{array}{l}\text { Lipolysis } \\
\text { of VLDL }\end{array}$ & $\begin{array}{l}\text { Deliver cholesterol } \\
\text { to cells }\end{array}$ & $22-28$ & $1.006-1.063$ & apoB100 & $\begin{array}{l}\sim 9 \% \text { that } \\
\text { in plasma }\end{array}$ & 10 & 50 & 29 & 11 & 20 \\
\hline HDL & $\begin{array}{c}\text { Liver and } \\
\text { ileum }\end{array}$ & $\begin{array}{c}\text { Reverse cholesterol } \\
\text { transport }\end{array}$ & $7-11$ & $1.063-1.21$ & apoA1 & $\begin{array}{l}\sim 20 \% \text { that } \\
\text { in plasma }\end{array}$ & 6 & 40 & 46 & 7 & 50 \\
\hline
\end{tabular}

TG, triglyceride; PL, phospholipid; Chol, cholesterol.

by the transendothelial transfer of HDL from plasma, indicating that HDLs acquire cholesterol from cells within the extravascular compartment. Pre $\beta$-HDL concentration in lymph was positively and independently related to both plasma pre $\beta$-HDL and lymph $\alpha$-HDL (mature, spheroidal cholesteryl ester-rich [CE-rich] HDL) concentrations, suggesting that lymph pre $\beta$-HDL particles result not only from transport out of plasma, but also from remodeling of plasma-derived $\alpha$-HDL in interstitial fluid (Figure 2). Concordant results were obtained when the same particles were quantified by chromatography (32). Subsequent in vitro incubation studies showed that lipoprotein remodeling in interstitial fluid generates pre $\beta$-HDL from $\alpha$-HDL, in contrast to plasma in which there is net conversion in the reverse direction (7). That the duration of these incubations was no greater than the apparent average residence time of HDL in the extracellular matrix in humans (33) suggests that this process may be an important source of pre $\beta$-HDLs in vivo.

By comparing cholesterol contents of size subclasses of HDLs in lymph with those in plasma, the rate of whole body cholesterol transport via lymph was estimated to average $0.89 \mathrm{mmol}$ (344 mg) per day, which was compatible with published estimates of whole body cholesterol turnover by isotope dilution analysis (34). Taken together, these studies suggest that the interstitium is a major site for generation of pre $\beta$-HDL, and implicate the lymphatic vasculature as the main transit route for movement of HDL from the interstitium to the bloodstream and liver. The concept that HDL relies on lymphatics to return to the blood during reverse cholesterol transport was demonstrated in mice, in which the lymphatic vasculature was surgically or genetically disrupted in skin, leading to marked reductions in the appearance of labeled cholesterol in plasma that originated from implanted tissue macrophages $(35,36)$.

Whether the number of HDL particles in interstitial fluid affects the rate of reverse cholesterol transport was assessed by intravenous infusion of reconstituted apoA1/lecithin discs, known to produce a rapid rise in plasma HDL concentration (37), into healthy humans previously given radiolabeled cholesterol (31). During seven days of continuous lymph collection, the infusion produced sequential increases in plasma HDLs, lymph pre $\beta$ HDLs, lymph cholesterol-specific radioactivity (consistent with efflux of cholesterol from tissues), and fecal bile acid excretion. No changes occurred in lymph concentrations of enzymes that collectively remodel HDL (38), including LCAT, which esterifies free cholesterol for packaging into the central core of spherical HDLs, or phospholipid transfer protein (PLTP) and CE transfer protein (CETP), which catalyze the exchange of lipids between HDL particles or to other lipoprotein subclasses. Indeed, cholesterol esterification rate and CETP activity are both very low in lymph compared with plasma (7). On the other hand, PLTP has a higher specific activity in lymph than in plasma, owing to a greater ratio of active to inactive forms (7). High specific activity of PLTP, along with absence of cholesterol esterification, which promotes conversion of pre $\beta$-HDL to $\alpha$-HDL, likely contributes to the propensity of interstitial fluid to generate pre $\beta$-HDL particles with a high activity for removing cholesterol from cells (7). In light of the need to better understand the impact of CETP inhibition on cholesterol transport in man (3), the effect of CETP activity or its inhibition on the efficiency with which HDL cycles through the interstitium is of paramount importance to investigate.

Overall, the collective evidence from these studies is consistent with the scheme in Figure 2. Passage of $\alpha$-HDLs into the interstitium across endothelium may occur by transcytosis through endothelial cells, as suggested in in vitro studies $(39,40)$, but ongoing work in vivo suggests a 2-pore model of ultrafiltration rather than transcytosis (our unpublished observations). When the known radii of different HDL subclasses are compared with those of the pores, the shift from small to large HDL subclasses that occurs when scavenger receptor B1 (SR-B1) and CETP are reduced would be expected to produce a major reduction in total $\alpha$-HDL transport into interstitial fluid, consistent with the observation that large lipoproteins do not enter the arterial wall (41). Small, lipid-poor apoA1-containing HDLs with pre $\beta$ electrophoretic mobility are generated in interstitial fluid by remodeling of spheroidal $\alpha$-HDLs derived from plasma. The pre $\beta$-HDLs in interstitial fluid then interact with ABCA1 transporters on extravascular cells to acquire unesterified cholesterol and phospholipid, resulting in formation of discoidal HDL, which travel to blood via the lymphatic system along with other macromolecules that exceed the radius of TNF- $\alpha$ $(3.24 \mathrm{~nm})(42)$. HDL ranges in radius from 3.82 to $5.43 \mathrm{~nm}(43,44)$. Upon re-entering blood at the thoracic duct, discoidal HDLs act as efficient substrates for LCAT, generating spheroidal $\alpha$-HDLs rich in CEs. Transfer of CEs to the liver occurs by two processes: direct uptake from $\alpha$-HDLs via SR-B1 and transfer to VLDLs and LDLs via CETP. The cyclical extravascular-intravascular remodeling of HDL is critical to maintaining a flow of cholesterol from peripheral cells to the liver for re-utilization and elimination as bile acids. 


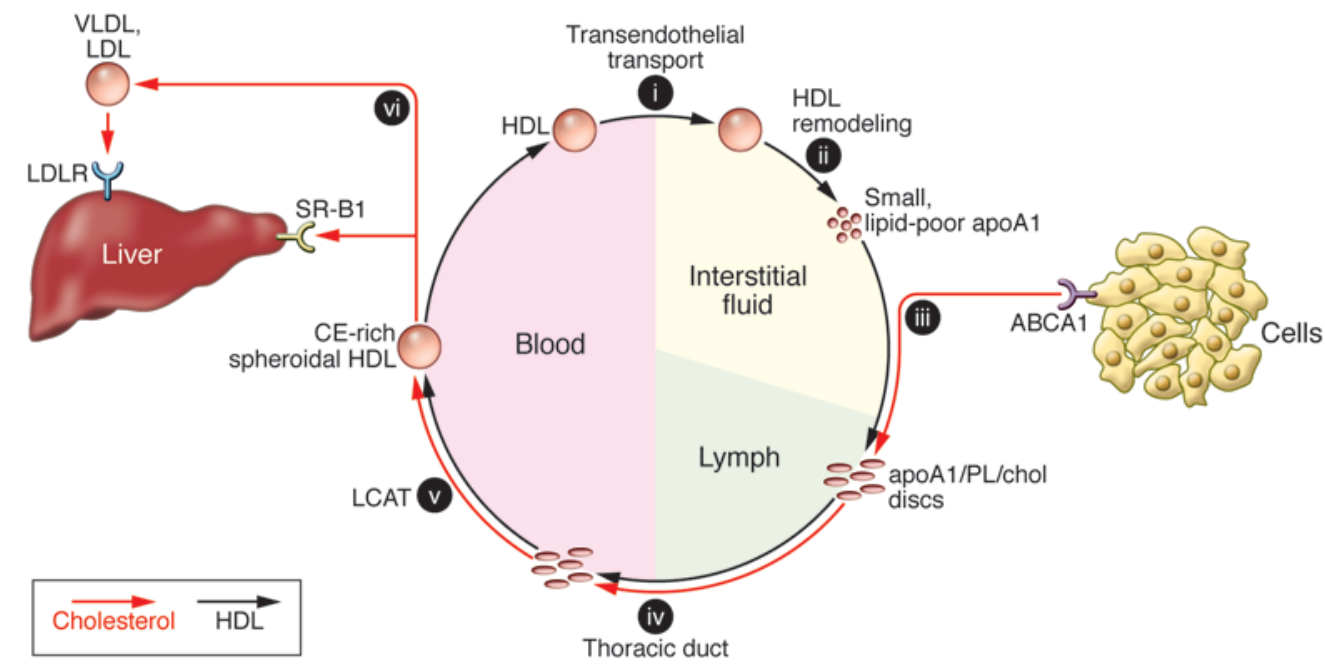

\section{Figure 2}

The intravascular/extravascular cycle of HDL remodeling that maintains reverse cholesterol transport. (i) Transfer of HDL across vascular endothelium. (ii) Production of small, lipid-poor apoA1-containing pre $\beta$-HDLs in interstitial fluid through the remodeling of spheroidal CE-rich $\alpha$-HDLs. (iii) Conversion of pre $\beta$-HDLs to discoidal HDLs through uptake of unesterified cholesterol (chol) and phospholipid (PL) via the ABCA1 transporters of peripheral cells. (iv) Transport of the discs via the lymphatic system to the blood via the thoracic duct. (v) Conversion of the discs to spheroidal CE-rich $\alpha$-HDLs in plasma through the action of LCAT. (vi) Transfer of CE from $\alpha$-HDLs to liver cells, directly via SR-B1 receptors and indirectly via CETP and apoB-containing lipoproteins (VLDLs and LDLs) that are endocytosed by apoB100 receptors. The principal function of LCAT is to generate CEs for delivery to the liver. The net production of pre $\beta$-HDLs in interstitial fluid appears to be maintained by a high ratio of active to inactive PLTP in the presence of a near-zero cholesterol esterification rate, in contrast to a high esterification rate and lower active/inactive PLTP ratio in plasma. Black arrows represent the path of apoA1 as a component of different HDLs as they move between the intravascular and extravascular compartments. Red arrows represent the flow of cholesterol, initially as unesterified cholesterol in interstitial fluid and lymph, and then as CE in blood.

\section{Lipoprotein entry into lymphatic vasculature: lessons from chylomicrons}

Peripheral lymph differs in function and composition from intestinal lymph. The latter transports newly synthesized chylomicrons, which are at least an order of magnitude greater in radius than HDL, from the ileum to the bloodstream during the absorption of ingested fat. Together with the liver, the ileum is a significant source of newly synthesized apoA1, which appears in intestinal lymph partly as a component of nascent chylomicrons and partly in combination with phospholipid and cholesterol as discoidal nascent HDL $(45,46)$. The absorptive lymphatic capillaries for lipoproteins in the intestine extend as a single lymphatic vessel in each villus, termed lacteals (Figure 1). These drain into mesenteric collecting lymphatic vessels that run through mesenteric fat and actively pump lymph under muscular and neural control (47-49). This lymph runs through mesenteric LNs and ultimately into the thoracic duct that drains the transported chylomicron-rich lymph into the bloodstream at the left subclavian vein. It is notable that this pattern of transport results in passage of lymph-derived lipoproteins, including the nutrients collected as "fatty meal," through not only mesenteric LNs (prior to entering the thoracic duct), but also through the heart and vasculature of the lung and subsequently other organs, where they are degraded by lipoprotein lipase. The remnant particles derived from these lipoproteins have access to the liver, strongly contrasting with non-fatty nutrients that directly enter the portal venous circulation from intestinal villi. One consequence of this route of transit is that the lung can be exposed to lipolysaccharides and other components from intestinal microbiota with an affinity for lipoproteins that enter lymph (50). Therefore, when gut leakage of microbiota is sufficiently great as to threaten organ failure, the lung is particularly susceptible (50). It is thus important that LNs, through which all lymph runs, respond to and filter absorbed lymph to protect the host against inflammatory lipoproteins. Mice lacking the lipoprotein lipase inhibitor angiopoietin-like 4 , which is expressed in mesenteric LN macrophages, are susceptible to lethal inflammation within mesenteric LNs exposed to chylomicrons bearing saturated fats (51). Secretion of apoA1 by the intestine itself may also protect the host from mesenteric inflammation, given the anti-inflammatory properties of HDL (52). It would be interesting to test whether adverse responses to gut leakage are heightened when apoA1 secretion by enterocytes is selectively lost.

Lymphatic vessels from all peripheral organs, like those from intestine, converge with the thoracic duct so that lymph delivered to the venous blood supply is a mixture of intestinal lymph and lymph draining other peripheral tissues (Figure 1). It remains uncertain how similar the mechanisms are for lipoprotein uptake between intestinal lacteals and lymphatic capillaries in other organs. We favor the concept that lipoproteins enter lymph, whether lacteals or lymphatic capillaries in other organs, by mechanisms that are nonselective with respect to molecular composition of the incoming cargo or specific receptors. This view, supported by the similarity between interstitial fluid and lymph (15), could explain how a variety of exogenous molecules, including tracer dyes, dextrans, foreign proteins, and nanoparticles, readily enter lymph. This view does not preclude the possibility that active mecha- 
nisms like macropinocytosis contribute to entry of fluid into the lymphatic vessel (17). In opposition to the concept of receptorindependent entry, Lim et al. reported that SR-B1 is required for the uptake of HDL in the skin lymphatic vasculature (36), implying that HDL could become trapped in tissues by loss of SR-B1 on lymphatic capillaries. If this finding is widely applicable, the premise that assessments of lipoproteins in afferent lymph mirror those in interstitial fluid may not hold under at least some circumstances. By contrast, the presence or absence of SR-B1 does not modulate chylomicron absorption in the intestine (53). Perhaps alternative explanations exist for the proposed role of SR-B1 in HDL transit out of the skin. For instance, the SR-B1-deficient mouse accumulates very large HDL particles in the circulation $(54,55)$, such that the plasma may be unable to supply the interstitium with HDL acceptors, leading to an alternative explanation for the failure of reverse cholesterol transport of exogenously administered cholesterol. Furthermore, SR-B1 plays a pivotal role in platelet function (56). Platelets are critical mediators in development of the lymphatic vasculature through their expression of CLEC2 (57-59), a key C-type lectin receptor for podoplanin that is widely expressed on lymphatic endothelial cells. Thus, the skin lymphatic vasculature may be abnormal in SR-B1-deficient mice, thereby negatively affecting reverse cholesterol transport without direct receptor-mediated uptake of HDL into lymphatics. On the other hand, as evidence indicates an extrahepatic role for SR-B1 in promoting cardiovascular disease (60), the possibility that either the uptake of HDL into tissue via vascular endothelium, which may at least partially require SR-B1 (61), or its egress from tissue via lymphatics (36) might contribute to poor reverse cholesterol transport, and therefore to atherosclerosis, is plausible and intriguing.

Though more is known about uptake of chylomicrons into lacteals than about uptake of lipoproteins into peripheral lymphatics, there is a paucity of literature overall in this important area. Newborn mice deficient in pleomorphic adenoma gene-like 2 (Plag2) succumb to a wasting syndrome stemming from failed chylomicron absorption (62). In this study, PLAG2 was highly expressed by enterocytes, with at least some expression by lacteals as well. Oil red O staining indicated accumulation of lipid within enterocytes, while electron micrographs revealed that chylomicrons were released from the epithelium but could not enter the lacteal. Plag2 deficiency also impeded the uptake of cholesterol into other tissues from the plasma. There has been little follow-up to this study, and it remains unclear whether chylomicron uptake is coordinated by lacteals in a PLAG2-dependent manner or if other changes related to chylomicron secretion, composition, or size account for the outcomes observed.

With regard to particle size, it is possible to develop a working model to explain why fats packaged in chylomicrons exclusively enter the lymphatic vasculature, whereas most nutrients traverse the portal venous system for primary delivery to the liver. The blood capillary network that surrounds each lacteal is fenestrated, particularly along the venous side. These fenestrations facilitate resorption of nutrients, even as they also allow ultrafiltration of molecules from the systemic vasculature into the lamina propria. However, the fenestrae are too narrow to permit passage of even the smaller size range of chylomicrons (63). Therefore, size exclusion, much like the mechanism postulated for HDL entry into skin lymphatics (42), likely accounts for why chylomicrons are directed to the periphery through the lymphatic vasculature, rather than to liver through the portal venous vasculature. The tip of the lacteal is thought to contain large pores that, in contrast to the nearby blood vessels, are sufficient in size to allow chylomicron entry (64). That the fenestrated blood vessels lie atop the lacteal around much of its exposed surface likely contributes to ensuring that smaller molecules, including hydrophilic nutrients and antigens not packaged in chylomicrons, primarily access the blood vasculature for transport, although there is a certain probability that a portion of these small molecules bypasses the fenestrated vasculature and enters the lymph (Figure 1), consistent with experimental observations. Furthermore, the rich macrophage and DC network in intestinal villi acquires many macromolecules that enter intestinal villi through robust endocytosis (65). Their collective endocytic activity protected the lacteal from absorption of tracer antigens, whereas depletion of these cells allowed increased absorption into the lacteal, with a resulting shift in the ensuing immune response (65). The study did not investigate whether the presence of macrophages and DCs affected chylomicron absorption or absorption of other nutrients. This issue deserves attention in future research because of its important implications. First, drugs engineered to target chylomicrons for transport into the lymphatic vasculature might avoid the liver toxicity sometimes associated with higher doses of drugs that are transported through the portal venous vasculature (66). On the other hand, the fact that environmental toxins like dichlorodiphenyltrichloroethane first gain access to the systemic circulation, rather than the portal circulation, where they could be detoxified by the liver, enhances the danger they pose to human health (67). Thus, for reasons ranging from maintenance of cardiovascular and immunologic health to the avoidance of drug toxicity, a better understanding is needed about how lymphatic vessels in the intestine absorb chylomicrons and other macromolecules. We believe these studies have merit in their own right and also provide a basis for future studies on HDL entry into lymphatics in the periphery.

\section{Atherosclerosis and cholesterol transport through interstitium and lymph}

Successful clinical interventions to improve cardiovascular health based on targeting HDL may require that we more thoroughly explore how the HDL cycle (Figure 2) is regulated and how it may differ in various tissues and organs. The propensity of macrophages to donate cholesterol to HDL during reverse cholesterol transport differs between different anatomical compartments (35). Yet the reason for this remains unclear. Is apoA1 more enriched in interstitial fluid at different anatomical sites? Or does the relative interstitial fluid space around macrophages influence reverse cholesterol transport, such that sites of inflammation, for example atherosclerotic plaque, where macrophages are aggregated would support a more sluggish rate of reverse cholesterol transport? Does the rate of interstitial fluid flow measurably influence the rate and extent of reverse cholesterol transport? Does the efficiency of lymph transport overall affect the development or reversibility of cholesterol-driven diseases like atherosclerosis?

Answering these questions requires more research on the role of lymphatics in clearing cholesterol from artery walls where atherosclerotic plaques occur. The adventitia of large arteries is supplied with lymphatic vasculature as part of the vasa vasorum, and advanced atherosclerotic plaques promote the growth of lymphatic vessels within the intima of plaques (68). Martel et al. employed a surgical technique that suggests lymphatic vessels mediate removal of cholesterol from the artery wall (35). This work, reviewed in greater detail elsewhere (69), needs to be verified in models that do not require lymphatic remodeling as part 
of the experiment. Achieving this goal will likely require experimental models with larger lymphatic vessels than are observed in mice. Experimental surgical interventions in the pig do not require full aortic transplant. Remarkably, delivery of labeled cholesterol esters, either in the form of LDL or HDL, to a ligated and temporarily bypassed segment of the pig thoracic aorta revealed that HDL passes through the media and enters adventitia efficiently, leading investigators in the late 1980s to conclude that HDL was likely cleared through adventitial lymphatics $(70,71)$. Labeled LDL, by contrast, penetrated only into the intimal layer, indicating specificity in trafficking through the medial wall for labeled HDL.

Nonetheless, the transport of HDL in arteries is less well studied and may differ from skin, so caution should be exercised in extrapolating from studies of peripheral lymph to the interstitial fluid of diseased arteries. Furthermore, much of the apoA1 in plaque has been rendered dysfunctional (72). On the other hand, as skin is the largest organ in the body, a substantial fraction of apoA1 is continually found there, making skin a key player in the HDL cycle regardless of how lipoproteins are transported from arteries. Indeed, hypercholesterolemic mice lacking apoA1 suffer from massive sequestration of cholesterol predominantly in skin (73). Hypercholesterolemia also impairs lymphatic transport from the skin (74), but quantifying transport from other body sites, including the artery wall, will require the development of novel assays.

\section{Conclusion}

Because it has proven more challenging than expected to understand the HDL cycle well enough to manipulate it therapeutically, significant attention should be focused on the half of apoA1bearing HDL particles found within interstitia. This part of the HDL life cycle remains relatively inaccessible for study, yet transit through the interstitium is certainly as critical as the period that HDL spends in plasma. Although recent studies have recognized that simple measurements of plasma HDL cholesterol are insufficient to predict efficacy in promoting cholesterol efflux, new assays to measure HDL function still focus on HDL in the plasma (64), making it impossible to determine whether some individuals have defective trafficking or activity of HDL within the interstitium. However, a critical unanswered question is whether evaluation of HDL remodeling and passage through skin, by far the largest and most accessible tissue, would be valuable or detract from our understanding of HDL in the interstitium of the artery wall. On the other hand, focusing on mechanisms that regulate passage of HDL through any interstitium, including those that enhance passage of nascent HDL into tissues and support its ability to later enter lymph loaded with large amounts of cholesterol, would likely benefit our understanding of cholesterol uptake by HDL in all tissues, including the artery wall.

Easton et al. infused reconstituted HDL and observed clearance of apoA1 from the plasma in a biphasic manner (75), with a secondary rise in apoA1 between 24 to 48 hours, consistent with two pools of $\operatorname{HDL}(75,76)$. The second pool is likely the interstitial pool of HDL, in which HDL has a mean residence time of approximately 29 hours (33). Thus, the second rise of apoA1 may mark the return of HDL to plasma after its transit through the interstitium, much of it likely in transit through the large organ of the skin. Nanjee et al. (31) observed a similar biphasic effect on plasma pre $\beta$-HDL concentration after intravenous infusion of reconstituted HDL, compatible with delayed appearance in plasma of pre $\beta$-HDLs generated from increased remodeling of plasma-derived $\alpha$-HDLs in the interstitium. Detailed assessment of this biphasic clearance is warranted. If it serves as a readout of interstitial passage of HDL, an assay more accessible than lymph cannulation may emerge to allow estimates of HDL flux through the interstitium in large cohorts of people. These assessments in turn would make it possible to determine whether such information provides valuable predictors for coronary health.

\section{Acknowledgments}

The authors are grateful for insightful discussions with Mary SorciThomas (Wake Forest University, Winston-Salem, North Carolina, USA) and Nick Davidson (Washington University School of Medicine, St. Louis, Missouri, USA) prior to the preparation of this article. The preparation of this article was supported in part by NIH grants HL096539 and AI049653 and a Breakthrough Award from the Kenneth Rainin Foundation to G.J. Randolph.

Address correspondence to: Gwendalyn J. Randolph, Department of Pathology and Immunology, Box 8118, Washington University School of Medicine, St. Louis, Missouri 63110, USA. Phone: 314.286.2345; Fax: 314.286.2362; E-mail: grandolph@path.wustl.edu. Or to: Norman E. Miller, Magdalen College, Oxford OX1 4AU, United Kingdom. Phone: 44.20.7490.3241; Fax: 44.20.7490.3241; E-mail: norman.miller@magd.ox.ac.uk.
1. Glomset JA, Janssen ET, Kennedy R, Dobbins J. Role of plasma lecithin:cholesterol acyltransferase in the metabolism of high density lipoproteins. J Lipid Res. 1966;7(5):638-648.

2. Weinstein DB, Carew TE, Steinberg D. Uptake and degradation of low density lipoprotein by swine arterial smoot muscle cells with inhibition of cholesterol biosynthesis. Biochim Biophys Acta. 1976; 424(3):404-421.

3. Barter PJ, Rye KA. Cholesteryl ester transfer protein inhibition as a strategy to reduce cardiovascular risk. J Lipid Res. 2012;53(9):1755-1766.

4. Schwartz GG, et al. Effects of dalcetrapib in patients with a recent acute coronary syndrome. N Engl J Med. 2012;367(22):2089-2099.

5 . Boden WE, et al. Niacin in patients with low HDL cholesterol levels receiving intensive statin therapy. NEngl J Med. 2011;365(24):2255-2267.

6. Voight BF, et al. Plasma HDL cholesterol and risk of myocardial infarction: a mendelian randomisation study. Lancet. 2012;380(9841):572-580.

7. Miller NE, et al. Lipoprotein remodeling generates lipid-poor apolipoprotein A-I particles in human interstitial fluid. Am J Physiol Endocrinol Metab. 2013;304(3):E321-E328.

8. Mora S, Otvos JD, Rifai N, Rosenson RS, Buring JE, Ridker PM. Lipoprotein particle profiles by nuclear magnetic resonance compared with standard lipids and apolipoproteins in predicting incident cardiovascular disease in women. Circulation. 2009; 119(7):931-939.

9. Nanjee MN, Cooke CJ, Olszewski WL, Miller NE. Lipid and apolipoprotein concentrations in prenodal leg lymph of fasted humans. Associations with plasma concentrations in normal subjects, lipoprotein lipase deficiency, and LCAT deficiency. J Lipid Res. 2000;41(8):1317-1327.

10. Krogstad AL, Jansson PA, Gisslen P, Lonnroth P. Microdialysis methodology for the measurement of dermal interstitial fluid in humans. BrJ Dermatol. 1996;134(6): 1005-1012.

11. Schmelz M, Luz O, Averbeck B, Bickel A. Plasma extravasation and neuropeptide release in human skin as measured by intradermal microdialysis.
Neurosci Lett. 1997;230(2):117-120.

12. Rutili G, Arfors KE. Collections of interstitial fluid: a comparison between wick and micropuncture techniques. Microvasc Res. 1978;15(1):107-109.

13. Duverger N, Ghalim N, Ailhaud G, Steinmetz A, Fruchart JC, Castro G. Characterization of apoAIV-containing lipoprotein particles isolated from human plasma and interstitial fluid. Arterioscler Thromb. 1993;13(1):126-132.

14. Kiistala U. Suction blister device for separation of viable epidermis from dermis. JInvest Dermatol. 1968; 50(2):129-137.

15. Sloop CH, Dory L, Roheim PS. Interstitial fluid lipoproteins. J Lipid Res. 1987;28(3):225-237.

16. Baluk P, et al. Functionally specialized junctions between endothelial cells of lymphatic vessels. JExp Med. 2007;204(10):2349-2362.

17. Dixon JB, Raghunathan S, Swartz MA. A tissueengineered model of the intestinal lacteal for evaluating lipid transport by lymphatics. Biotechnol Bioeng. 2009;103(6):1224-1235.

18. Reichl D, Pflug JJ. The concentration of 
apolipoprotein A-I in human peripheral lymph. Biochim Biophys Acta. 1982;710(3):456-463.

19. Reichl D, Rudra DN, Pflug J. The concentration of apolipoprotein A-II in human peripheral lymph. Biochim Biophys Acta. 1989;1006(2):246-249.

20. Reichl D, Myant NB, Pflug JJ. Concentration of lipoproteins containing apolipoprotein B in human peripheral lymph. Biochim Biophys Acta. 1977; 489(1):98-105.

21. Hong JL, Pflug J, Reichl D. Comparison of apoprotein B of low density lipoproteins of human interstitial fluid and plasma. Biochem J. 1984;222(1):49-55.

22. Reichl D, Postiglione A, Myant NB, Pflug JJ, Press $\mathrm{M}$. Observations on the passage of apoproteins from plasma lipoproteins into peripheral lymph in two men. Clin Sci Mol Med. 1975;49(5):419-426.

23. Reichl D, Simons LA, Myant NB, Pflug JJ, Mills GL. The lipids and lipoproteins of human peripheral lymph, with observations on the transport of cholesterol from plasma and tissues into lymph. Clin Sci Mol Med. 1973;45(3):313-329.

24. Rudra DN, Myant NB, Pflug JJ, Reichl D. The distribution of cholesterol and apoprotein A-I between the lipoproteins in plasma and peripheral lymph from normal human subjects. Atherosclerosis. 1984;53(3):297-308.

25. Reichl D, Miller NE. The anatomy and physiology of reverse cholesterol transport. Clin Sci (Lond). 1986;70(3):221-231.

26. Reichl D, Hathaway CB, Sterchi JM, Miller NE. Lipoproteins of human peripheral lymph. Apolipoprotein AI-containing lipoprotein with alpha-2 electrophoretic mobility. Eur J Clin Invest. 1991;21(6):638-643.

27. Castro GR, Fielding CJ. Early incorporation of cell-derived cholesterol into pre- $\beta$-migrating highdensity lipoprotein. Biochemistry. 1988;27(1):25-29.

28. Reichl D, Miller NE, Sterchi JM. Gemfibrozil increases apolipoprotein A-I and cholesterol concentrations in human peripheral lymph. Eur J Clin Invest. 1993;23(4):254-258.

29. Nanjee MN, Cooke CJ, Wong JS, Hamilton RL, Olszewski WL, Miller NE. Composition and ultrastructure of size subclasses of normal human peripheral lymph lipoproteins: quantification of cholesterol uptake by HDL in tissue fluids. J Lipid Res. 2001;42(4):639-648.

30. Engeset A, Hager B, Nesheim A, Kolbenstvedt A. Studies on human peripheral lymph. I. Sampling method. Lymphology. 1973;6(1):1-5.

31. Nanjee MN, et al. Intravenous apoA-I/lecithin discs increase pre-beta-HDL concentration in tissue fluid and stimulate reverse cholesterol transport in humans. J Lipid Res. 2001;42(10):1586-1593.

32. Nanjee MN, Cooke CJ, Olszewski WL, Miller NE. Concentrations of electrophoretic and size subclasses of apolipoprotein A-I-containing particles in human peripheral lymph. Arterioscler Thromb Vasc Biol. 2000;20(9):2148-2155.

33. Hovorka R, Nanjee MN, Cooke CJ, Miller IP, Olszewski WL, Miller NE. Mass kinetics of apolipoprotein A-I in interstitial fluid after administration of intravenous apolipoprotein A-I/lecithin discs in humans. J Lipid Res. 2006;47(5):975-981.

34. Miller NE, Nestel PJ, Clifton-Bligh P. Relationships between plasma lipoprotein cholesterol concentrations and the pool size and metabolism of cholesterol in man. Atherosclerosis. 1976;23(3):535-547.

35. Martel C, et al. Lymphatic vasculature mediates macrophage reverse cholesterol transport in mice. J Clin Invest. 2013;123(4):1571-1579.

36. Lim HY, et al. Lymphatic vessels are essential for the removal of cholesterol from peripheral tissues by SR-BI-mediated transport of HDL. Cell Metab. 2013;17(5):671-684.

37. Nanjee MN, Doran JE, Lerch PG, Miller NE. Acute effects of intravenous infusion of ApoA1/phosphatidylcholine discs on plasma lipoproteins in humans. Arterioscler Thromb Vasc Biol. 1999; 19(4):979-989.

38. Kujiraoka T, et al. Effects of intravenous apolipoprotein A-I/phosphatidylcholine discs on LCAT, PLTP, and CETP in plasma and peripheral lymph in humans. Arterioscler Thromb Vasc Biol. 2003; 23(9):1653-1659.

39. Rohrer L, Cavelier C, Fuchs S, Schluter MA, Volker W, von Eckardstein A. Binding, internalization and transport of apolipoprotein A-I by vascular endothelial cells. Biochim Biophys Acta. 2006;1761(2):186-194.

40. Cavelier C, Ohnsorg PM, Rohrer L, von Eckardstein A. The beta-chain of cell surface $F(0) F(1)$ ATPase modulates apoA-I and HDL transcytosis through aortic endothelial cells. Arterioscler Thromb Vasc Biol. 2012;32(1):131-139.

41. Nordestgaard BG, Stender S, Kjeldsen K. Reduced atherogenesis in cholesterol-fed diabetic rabbits. Giant lipoproteins do not enter the arterial wall. Arteriosclerosis. 1988;8(4):421-428.

42. Miller NE, et al. Secretion of adipokines by human adipose tissue in vivo: partitioning between capillary and lymphatic transport. Am J Physiol Endocrinol Metab. 2011;301(4):E659-E667.

43. Blanche PJ, Gong EL, Forte TM, Nichols AV. Characterization of human high-density lipoproteins by gradient gel electrophoresis. Biochim Biophysi Acta. 1981;665(3):408-419.

44. Cheung MC, Albers JJ. Characterization of lipoprotein particles isolated by immunoaffinity chromatography. Particles containing A-I and A-II and particles containing A-I but no A-II. J Biol Chem. 1984;259(19):12201-12209.

45. Alaupovic P, Furman RH, Falor WH, Sullivan ML, Walraven SL, Olson AC. Isolation and characterization of human chyle chylomicrons and lipoproteins. Ann N Y Acad Sci. 1968;149(2):791-807.

46. Kostner G, Holasek A. Characterization and quantitation of the apolipoproteins from human chyle chylomicrons. Biochemistry. 1972; 11(7):1217-1223.

47. Schmid-Schonbein GW. Microlymphatics and lymph flow. Physiol Rev. 1990;70(4):987-1028.

48. Muthuchamy M, Zawieja D. Molecular regulation of lymphatic contractility. Ann N Y Acad Sci. 2008;1131:89-99.

49. Dixon JB. Mechanisms of chylomicron uptake into lacteals. Ann N Y Acad Sci. 2010; 1207(suppl 1):E52-E57.

50. Deitch EA. Gut lymph and lymphatics: a source of factors leading to organ injury and dysfunction. Ann N Y Acad Sci. 2010;1207(suppl 1):E103-E111.

51. Lichtenstein L, et al. Angptl4 protects against severe proinflammatory effects of saturated fat by inhibiting fatty acid uptake into mesenteric lymph node macrophages. Cell Metab. 2010;12(6):580-592.

52. Navab M, et al. High-density lipoprotein and $4 \mathrm{~F}$ peptide reduce systemic inflammation by modulating intestinal oxidized lipid metabolism: novel hypotheses and review of literature. Arterioscler Thromb Vasc Biol. 2012;32(11):2553-2560.

53. Bura KS, et al. Intestinal SR-BI does not impact cholesterol absorption or transintestinal cholesterol efflux in mice. J Lipid Res. 2013;54(6):1567-1577.

54. Rigotti A, Trigatti BL, Penman M, Rayburn H, Herz $\mathrm{J}$, Krieger M. A targeted mutation in the murine gene encoding the high density lipoprotein (HDL) receptor scavenger receptor class B type I reveals its key role in HDL metabolism. Proc Natl Acad Sci US A. 1997;94(23):12610-12615.

55. Varban ML, et al. Targeted mutation reveals a central role for SR-BI in hepatic selective uptake of high density lipoprotein cholesterol. Proc Natl Acad Sci U S A. 1998;95(8):4619-4624.

56. Nofer JR, van Eck M. HDL scavenger receptor class B type I and platelet function. Curr Opin Lipidol. 2011;22(4):277-282.
57. Bertozzi CC, Hess PR, Kahn ML. Platelets: covert regulators of lymphatic development. Arterioscler Thromb Vasc Biol. 2010;30(12):2368-2371.

58. Uhrin P, et al. Novel function for blood platelets and podoplanin in developmental separation of blood and lymphatic circulation. Blood. 2010;115(19):3997-4005.

59. Carramolino L, Fuentes J, Garcia-Andres C, Azcoitia V, Riethmacher D, Torres M. Platelets play an essential role in separating the blood and lymphatic vasculatures during embryonic angiogenesis. Circ Res. 2010;106(7):1197-1201.

60. Huby T, et al. Knockdown expression and hepatic deficiency reveal an atheroprotective role for SR-BI in liver and peripheral tissues. J Clin Invest. 2006;116(10):2767-2776.

61. Ohnsorg PM, et al. Carboxyl terminus of apolipoprotein A-I (ApoA-I) is necessary for the transport of lipid-free ApoA-I but not prelipidated ApoA-I particles through aortic endothelial cells. J Bio Chem. 2011;286(10):7744-7754.

62. Van Dyck F, et al. Loss of the PlagL2 transcription factor affects lacteal uptake of chylomicrons. Cell Metab. 2007;6(5):406-413.

63. Braet F, Wisse E. Structural and functional aspects of liver sinusoidal endothelial cell fenestrae: a review. Comp Hepatol. 2002;1(1):1.

64. Lee JS. Tissue fluid pressure, lymph pressure, and fluid transport in rat intestinal villi. Microvasc Res. 1986;31(2):170-183.

65. Chang SY, et al. Circulatory antigen processing by mucosal dendritic cells controls CD8 $(+)$ T cell activation. Immunity. 2013;38(1):153-165.

66. Porter CJ, Trevaskis NL, Charman WN. Lipids and lipid-based formulations: optimizing the oral delivery of lipophilic drugs. Nat Rev Drug Discov. 2007;6(3):231-248.

67. Kohan AB, Vandersall AE, Yang Q, Xu M, Jandacek RJ, Tso P. The transport of DDT from chylomicrons to adipocytes does not mimic triacylglycerol transport. Biochim Biophys Acta. 2013;1831(2):300-305.

68. Kholova I, et al. Lymphatic vasculature is increased in heart valves, ischaemic and inflamed hearts and in cholesterol-rich and calcified atherosclerotic lesions. Eur J Clin Invest. 2010;41(5):487-497.

69. Martel C, Randolph GJ. Atherosclerosis and transit of HDL through the lymphatic vasculature. Curr Atheroscler Rep. 2013;15(9):354.

70. Nordestgaard BG, Hjelms E, Stender S, Kjeldsen K. Different efflux pathways for high and low density lipoproteins from porcine aortic intima. Arteriosclerosis. 1990;10(3):477-485.

71. Hjelms E, Nordestgaard BG, Stender S, Kjeldsen K. A surgical model to study in vivo efflux of cholesterol from porcine aorta. Evidence for cholesteryl ester transfer through the aortic wall. Atherosclerosis. 1989;77(2-3):239-249.

72. DiDonato JA, et al. Function and distribution of apolipoprotein A1 in the artery wall are markedly distinct from those in plasma. Circulation. 2013;128(15):1644-1655

73. Zabalawi $\mathrm{M}$, et al. Inflammation and skin cholesterol in LDLr-/-, apoA-I-/- mice: link between cholesterol homeostasis and self-tolerance? J Lipid Res. 2007;48(1):52-65.

74. Lim HY, et al. Hypercholesterolemic mice exhibit lymphatic vessel dysfunction and degeneration. Am J Pathol. 2009;175(3):1328-1337.

75. Easton R, Gille A, D’Andrea D, Davis R, Wright SD, Shear C. A multiple ascending dose study of CSL112, an infused formulation of ApoA-I [published online ahead of print October 3, 2013]. J Clin Pharmacol. doi:10.1002/jcph.194.

76. Fidge N, Nestel P, Ishikawa T, Reardon M, Billington T. Turnover of apoproteins A-I and A-II of high density lipoprotein and the relationship to other lipoproteins in normal and hyperlipidemic individuals. Metabolism. 1980;29(7):643-653. 